\title{
Complemento de verbos de modalidade, manipulação e de cognição-elocução em Xikrín do Cateté
}

\author{
Complements of modality, manipulation \\ and cognition-elocution verbs in Xikrín do Cateté
}

Lucivaldo Silva da Costa ${ }^{1}$

\begin{abstract}
Resumo
Neste estudo descrevemos o que corresponde em Xikrin a orações dependentes ou subordinadas completivas em outras línguas do mundo, tendo como foco a investigação de sua natureza verbal ou nominal e os fatores que condicionam uma ou outra. Assim, iniciamos o estudo com uma breve introdução sobre as estratégias que as línguas naturais dispõem para expressar a subordinação de orações. Em seguida, discorremos sobre as estruturas correspondentes da língua Xikrín.
\end{abstract}

Palavras-chave: Língua Xikrín. Subordinação. Coordenação. Orações completivas. Nominalizações

\begin{abstract}
In this paper we describe what corresponds in Xikrín to dependent or subordinate completive clauses in other world languages, focusing on the investigation of its nature - verbal or nominal -, as well as on the factors motivating them. We start with a brief introduction about strategies displayed by natural languages to express subordinate clauses, which is followed by a description of the corresponding structures found in Xikrín.
\end{abstract}

Keywords: Xikrín. Subordonation. Coordination. Completive clauses. Nominalizations.

\section{Considerações iniciais}

A língua Xikrín é uma variedade do Kayapó (Rodrigues, 1986) falada tanto pelo povo Xikrín da Terra Indígena Xikrín do Cateté, localizada no município de Parauapebas, sudeste do estado do Pará, quanto pelos Xikrín do Bacajá, da Terra Indígena Trincheira Bacajá, que faz fronteira, a leste, com as rodovias Belém-Brasília (BR-010) e PA-150, na altura de Redenção e, a sudoeste, limitase com a sede do município de São Felix do Xingu². De acordo com o censo

${ }^{1}$ Doutor pela Universidade de Brasília. Professor Adjunto na Universidade Federal do Sul e Sudeste do Pará. lucivaldosc@unifesspa.edu.br

${ }^{2}$ As informações sobre os limites da TI Trincheira Bacajá foram retiradas do site: http:// 
da de 2012 da DSEI Altamira (Beltrame, comunicação pessoal), os Xikrín do Bacajá somam aproximadamente 806 indivíduos. Os Xikrín do Cateté vivem atualmente em três aldeias, a aldeia Cateté, maior e mais antiga, tem aproximadamente 800 indivíduos, a aldeia Djudjêkô tem aproximadamente 250 indivíduos, e a aldeia Ô'ôdjãm, a mais recente, tem aproximadamente 150 habitantes.

Nas seções seguintes tratamos de subordinação, em uma perspectiva tipológica e, em seguida, descrevemos as construções correspondentes em Xikrín.

\section{Algumas considerações sobre tipos de subordinação}

Thompson \& Longacre (1985: 172) distinguem três tipos de orações subordinadas: as completivas - que funcionam como sintagmas nominais, as relativas - que funcionam como modificadores de nomes, e as adverbiais - que funcionam como modificadores de verbos ou de toda a oração. Ainda, conforme Thompson \& Longacre (1985: 173), há três mecanismos atestados nas línguas do mundo usados para marcar orações subordinadas:

(a) Morfemas subordinadores, que podem ser de dois tipos: (i) morfemas gramaticais que não apresentam significado lexical, como to do inglês e (ii) morfemas gramaticais com conteúdo lexical, como before, when, if do inglês;

(b) Formas verbais especiais, isto é, aquelas que não são usadas em asserções independentes. Em línguas com concordância verbo-sujeito, a forma verbal especial pode ser a forma nãofinita que perde uma ou mais das categorias de concordância;

(c) Ordem de palavras. Algumas línguas têm uma ordem de palavras especial para orações subordinadas, como em alemão e em sueco.

Além das três estratégias citadas, Noonan (2007) afirma que a parataxe é também uma estratégia usada para expressar a subordinação de orações. Neste tipo de construção, tanto oração principal quanto oração dependente pode ser considerada como orações independentes, cada uma contendo sintagmas verbais com verbo flexionado, sem nenhum marcador de coordenação ou subordinação ligando as duas orações e sem nenhuma forma verbal especial. O quadro a seguir é uma síntese de como Noonan (1985: 65) aborda a complementação por meio da parataxe:

cggamgati.funai.gov.br/index.php/experiencias-em-gestao/terra-indigena-trincheirabacaja/, no dia 26 de maio de 2015. Acesso às 17:24h. 


\section{Quadro 01 - Complementação por parataxe}

\begin{tabular}{|c|c|c|c|c|}
\hline $\begin{array}{c}\text { Tipo de } \\
\text { complemento }\end{array}$ & $\begin{array}{c}\text { Classe de } \\
\text { palavra do } \\
\text { predicado }\end{array}$ & $\begin{array}{c}\text { Relação sintática } \\
\text { do sujeito com o } \\
\text { predicado }\end{array}$ & $\begin{array}{c}\text { Categorias } \\
\text { flexionais }\end{array}$ & $\begin{array}{c}\text { Outras } \\
\text { propriedades }\end{array}$ \\
\hline Paratático & verbo & $\begin{array}{c}\text { o predicado pode } \\
\text { concordar com } \\
\text { o sujeito, mas } \\
\text { não forma um } \\
\text { constituinte } \\
\text { com ele }\end{array}$ & $\begin{array}{c}\text { as mesmas do } \\
\text { indicativo }\end{array}$ & $\begin{array}{c}\text { asterpretado como separada; } \\
\text { sintaticamente } \\
\text { não é uma oração } \\
\text { subordinada; } \\
\text { não possui } \\
\text { complementizador }\end{array}$ \\
\hline
\end{tabular}

$\mathrm{Na}$ seção seguinte, apresentamos algumas características universais das orações completivas e, em seguida, mostramos como a língua Xikrín constrói estruturas correspondentes.

\section{Orações completivas}

Orações completivas funcionam sintaticamente como argumento do verbo da oração principal. Essa característica as distingue de outras orações subordinadas, como as relativas e as adverbiais (Noonan, 1985; Givón, 2001; Dixon, 2010).

Para Givón (2001: 40), os verbos que exigem complemento oracional agrupam-se em três classes semânticas principais:

- Verbos de modalidade ('querer', 'começar', 'terminar', 'tentar' etc.);

- Verbo de manipulação ('fazer', 'dizer', 'ordenar', 'pedir' etc.)

- Verbos de cognição-elocução ('ver', 'saber', 'pensar', etc.).

Nas subseções seguintes, descrevemos as propriedades de cada classe semântica segundo Givón e passamos à análise dos dados da língua Xikrín.

\subsection{Verbo de modalidade}

De acordo com Givón (2001: 55), orações principais que têm como núcleo verbos de modalidade codificam ações, estados ou atitudes modais (tentativa, intenção, obrigação, habilidade, possibilidade) e aspectuais (início, término, continuação, sucesso, fracasso) do sujeito frente ao evento/estado codificado na oração completiva.

Do ponto de vista sintático, a relação entre verbos modais e seus complementos são as seguintes (Givón, 2001:55): 
- O sujeito da oração principal é também o sujeito da oração completiva;

- O sujeito da oração completiva tem codificação zero;

- O verbo da oração completiva é comumente não-finito ou nominalizado;

- A oração completiva é análoga ao objeto da oração principal (OV ou VO);

- A oração completiva tende a ter um contorno intonacional unificado com a oração principal.

Pertencem à classe de verbos de modalidade temas como 'querer', 'começar', 'terminar', 'tentar' 'deixar', 'dever', 'poder', 'permitir', 'conseguir'.

Descrevemos, na seção seguinte, as estratégias da língua Xikrín para expressar o que em outras línguas, como o Inglês e o Português são orações completivas de verbos como 'terminar', 'começar', 'conseguir' e 'querer'.

\subsection{1 -ojnore 'terminar'}

Em Xikrín, o que corresponde a orações completivas do verbo 'terminar' em Inglês são nominalizações, como mostram os seguintes exemplos:

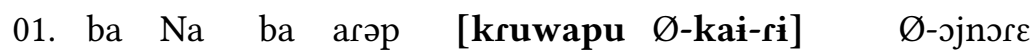

1 RLS 1 Já capacete $\mathbf{R}^{1}$-tecer-NLZ $\mathbf{R}^{1}$-terminar 'eu já terminei o tecer do capacete' '(eu já terminei de tecer o capacete)'

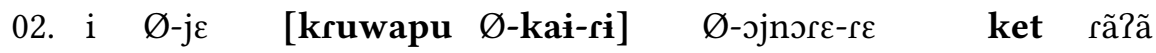
$1 \mathrm{R}^{1}$-OBL capacete $\mathrm{R}^{1}$-tecer-NLZ $\mathrm{R}^{1}$-terminar-NLZ NEG Ainda 'não houve ainda o terminar do tecer do capacete por mim' '(eu ainda não terminei de tecer o capacete)'

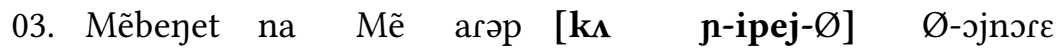
Velho RLS HUM Já paneiro $\mathrm{R}^{1}$-fazer-NLZ $\mathrm{R}^{1}$-terminar 'o velho já terminou o fazer do paneiro'

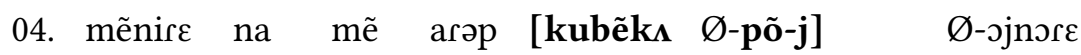
Mulher RLS HUM já roupa $\mathrm{R}^{1}$-lavar-NLZ $\mathrm{R}^{1}$-terminar 'a mulher já terminou o lavar de roupas' '(a mulher já terminou de lavar roupa)'

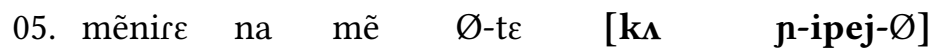
Mulher RLS HUM $\mathrm{R}^{1}$-OBL paneiro $\mathrm{R}^{1}$-fazer-NLZ 'a mulher já terminou o fazer do paneiro' '(a mulher já terminou de fazer o paneiro 


\subsection{2 -mokraj 'começar'}

O mesmo tipo de nominalização é usado como complemento do verbo 'começar' e dos verbos 'conseguir' e 'querer'.

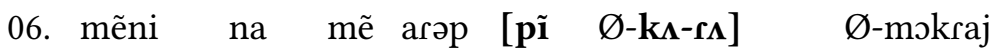
mulher RLS PL Já pau $\mathrm{R}^{1}$-cortar-NLZ $\mathrm{R}^{1}$-começar 'as mulheres já começaram o cortar do pau' '(as mulheres já começaram a cortar pau)'

07. mẽni na mẽ $\varnothing-t \varepsilon \quad\left[\begin{array}{ll}\tilde{\mathbf{i}} & \varnothing-\mathbf{k \Lambda}-\mathbf{r \Lambda}\end{array}\right]$

mulher RLS PL $\mathrm{R}^{1}$-OBL pau $\mathrm{R}^{1}$-cortar-NLZ

$\varnothing$-mokraj-Ø ket rã?ã

$\mathrm{R}^{1}$-começar-NLZ NEG ainda

'ainda não houve o começar de cortar pau pelas mulheres'

'(as mulheres ainda não começaram a cortar pau)'

08. I $\varnothing$-prõ dुa [mri n-ĩ

$1 \mathrm{R}^{1}$-esposa IRLS caça $\mathrm{R}^{1}$-carne $\mathrm{R}^{1}$-assar-NLZ $\mathrm{R}^{1}$-começar 'minha esposa começou o assar da carne de caça' '(minha esposa começou o assar de carne de caça)'

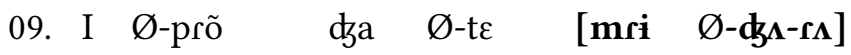

$1 \mathrm{R}^{1}$-esposa IRLS $\mathrm{R}^{1}$ - OBL caça $\mathrm{R}^{1}$-assar-NLZ

Ø-mokraj-Ø Ket rã?ã

$\mathrm{R}^{1}$-começar-NLZ NEG ainda

'não houve o começar do assar da caça por minha esposa' '(minha esposa não começou a assar a caça)'

10. pikadywa na arəp $\left[\begin{array}{lll}k \Lambda j & d y-w a y r \Lambda-j\end{array}\right] \quad \varnothing$-məkraj Pykadjwá RLS já facão $\mathrm{R}^{1}$-amolar-NLZ $\mathrm{R}^{1}$-começar

'Pykadjwá já começou o amolar do facão'

'(Pykadjwá já começou a amolar o facão)'

\section{1 boj 'conseguir'}

11. ba Na ba $[\mathbf{i} \varnothing$-je a $\varnothing$-mã

$1 \quad$ RLS $\quad 1 \quad 1 \quad \mathrm{R}^{1}$-OBL $\quad 2 \quad \mathrm{R}^{1}$-DIRET 
$\varnothing$-akre-Ø $\quad$ Ø-әгə] boj

$\mathrm{R}^{1}$-ensinar-NLZ $\quad \mathrm{R}^{1}$-DIR $\quad$ conseguir

'eu consegui com respeito ao ensinar para vocề' '(eu consegui te ensinar)'

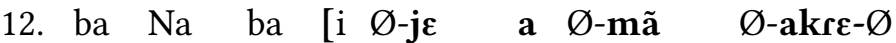

$1 \quad$ RLS $\quad 1 \quad 1 \quad \mathrm{R}^{1}$-OBL $\quad 2 \quad \mathrm{R}^{1}$-DIRET $\quad \mathrm{R}^{1}$-ensinar-NLZ

Ø-әгə] I Ø-boj-Ø $\quad$ ket rãrã

$\mathrm{R}^{1}$-DIR $\quad 1 \quad \mathrm{R}^{1}$-conseguir-NLZ NEG ainda

'não houve o conseguir na direção do ensinar a você por mim'

'(eu não consegui te ensinar)'

13. mẽmi $\mathrm{Na}$ mẽ [Ø-te ayro Ø-bĩ-n

homem RLS HUM $\mathrm{R}^{2}$-OBL porcão $\mathrm{R}^{1}$-matar-NLZ $\mathrm{R}^{1}$-DIR

Ø-boj-Ø Ket rã7ã

$\mathrm{R}^{1}$-conseguir-NLZ NEG ainda

'não houve o conseguir na direção do matar do porcão pelo homem'

'(o homem não conseguiu matar o porcão)'

14. ga $\mathrm{Na}$ ga $\left[\begin{array}{llll}\mathbf{a} & \varnothing-\mathbf{j} \boldsymbol{\varepsilon} & \mathbf{n i} & \varnothing-\mathbf{m} \tilde{\mathbf{u}}-\mathbf{j}\end{array}\right.$

$\begin{array}{lllllll}2 & \mathrm{RLS} & 2 & 2 & \mathrm{R}^{2} \text {-OBL } & \text { mulher } & \mathrm{R}^{1} \text {-ver-NLZ }\end{array}$

Ø-әгə] $\varnothing$-boj

$\mathrm{R}^{1}$-DIR $\mathrm{R}^{1}$-conseguir

'você conseguiu na direção do ver da mulher por você'

'(você conseguiu ver a mulher)'

15. ga $\mathrm{Na}$ ga $\left[\begin{array}{lllll}\mathbf{a} & \varnothing-\mathbf{j} \boldsymbol{\varepsilon} & \mathbf{n i} & \varnothing-\mathbf{m} \tilde{\mathbf{u}} \mathbf{j}\end{array}\right.$

2 RLS $2 \quad 2 \quad \mathrm{R}^{2}$-OBL mulher $\mathrm{R}^{1}$-ver-NLZ

Ø-әгә] А Ø-bој-

$\mathrm{R}^{1}$-DIR $\quad 2 \quad \mathrm{R}^{1}$-conseguir-NLZ NEG

'não houve o conseguir na direção do ver da mulher por você'

'(você não conseguiu ver a mulher)'

\subsection{4. prãm 'querer'}

16. ba na ba i Ø-mã

$\begin{array}{lllll}1 & \text { RLS } & 1 & 1 & \mathrm{R}^{1} \text {-DIRET }\end{array}$ 
[puru Ø-kare-re] $\quad$-prãm

Roça $\quad \mathrm{R}^{1}$-limpar-NLZ $\mathrm{R}^{1}$-querer

'eu quero o limpar a roça'

17. ba na ba i $\varnothing$-mã

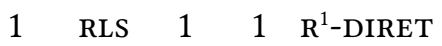

[puru Ø-kare-re] $\varnothing$-prãm ket

roça $\mathrm{R}^{1}$-limpar-NLZ $\mathrm{R}^{1}$-querer $\quad$ NEG

'eu não quero limpar a roça'

18. Kubẽ ku-mã [kaprãn Ø-krẽ-n] Ø-prãm não indígena $\quad \mathrm{R}^{2}$-DIRET jabuti $\quad \mathrm{R}^{1}$-comer-NLZ $\mathrm{R}^{1}$-querer

'o não indígena quer comer jabuti'

19. i $\varnothing$-mã tep j-anĩ-j $\quad$-prãm

$1 \mathrm{R}^{1}$-DIRET peixe $\mathrm{R}^{1}$-pescar-NLZ $\mathrm{R}^{1}$-querer 'eu quero pescar'

20. i $\varnothing$-mã tep j-anĩ-j $\quad$-prãm ket

1 R ${ }^{1}$-DIRET peixe $\mathrm{R}^{1}$-pescar-NLZ $\mathrm{R}^{1}$-querer NEG 'eu não quero pescar'

\subsection{Verbos de manipulação}

Orações principais que têm como núcleo verbos de manipulação apresentam as seguintes características semânticas e sintáticas, de acordo com Givón (2001: 41):

Características semânticas

- O agente da oração principal manipula o comportamento do manipulee 'alvo', um agente em potencial;

- O manipulee do verbo principal é correferente com o agente da oração completiva;

- A oração completiva codifica o evento a ser realizado pelo manipulee.

\section{Características sintáticas}

- O agente manipulador do verbo principal é o sujeito da oração principal; 
- O manipulee do verbo principal exerce a função de objeto direto ou indireto da oração principal;

- O manipulee do verbo principal é também o sujeito da oração completiva.

- Sujeito 'manipulee' da oração completiva é codificado como zero na oração completiva.

- O verbo da oração completiva exibe com maior frequência morfologia nominalizada ou não-finita.

- A oração completiva tende a ocupar a posição de objeto na oração principal (OV ou VO).

- A oração completiva tende a ter um contorno intonacional unificado com a oração principal.

São considerados de manipulação verbos como: 'fazer', 'dizer', 'ordenar', 'pedir', 'mandar', falar', dentre outros.

\subsubsection{Orações completivas com o verbo -arẽ 'dizer'}

As orações completivas com verbo de manipulação são sintaticamente independentes da oração matriz, mas completam o significado desta. Ocorrem justapostas, têm como núcleo do predicado formas verbais plenas e seu argumento sujeito é codificado pelas marcas pessoais nominativas.

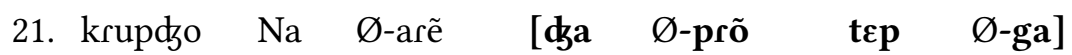
Krupdjo RLS $\mathrm{R}^{2}$-dizer IRRLS $\mathrm{R}^{2}$-esposa peixe $\mathrm{R}^{1}$-assar

'Krupdjo disse (que) (há potencialidade de que) esposa assa peixe'

22. Mẽni na mẽØ-arẽ [dga mẽ A

Mulher RLS HUM $\mathrm{R}^{2}$-dizerIRRLS $\quad$ PL 2

kwara d-une-j Ket

Mandioca $\quad \mathrm{R}^{2}$-espremer-NLZ NEG

'a mulher disse que (potencialmente) vocês não vão espremer mandioca'

23. ga $\mathrm{Na}$ ga i Ø-mã karino i j-arẽ

2 RLS $2 \quad 1 \quad \mathrm{R}^{1}$-DIRET fumo $1 \mathrm{R}^{1}$-dizer

[daa Ba a $\quad \varnothing$-mã ku-bi

$\begin{array}{lllll}\text { IRRLS } & 2 & 2 & \mathbf{R}^{1} \text {-DIRET } & \mathbf{R}^{2} \text {-pegar }\end{array}$

'você disse que (potencialmente) eu vou pegar fumo para você' 
24. ga $\mathrm{Na}$ ga i $\varnothing$-mã karino i j-arẽ

2 RLS $2 \quad 1 \quad \mathrm{R}^{1}$-DIRET fumo $1 \mathrm{R}^{1}$-dizer

$\left[\begin{array}{llllll}\text { dga } & \mathbf{I} & \varnothing-\mathbf{j} \varepsilon & \mathbf{a} & \varnothing-\mathbf{m a ̃} \quad \mathbf{k u}-\mathbf{b i}]\end{array}\right.$

IRRLS $\quad 1 \quad \mathrm{R}^{1}$-OBL $2 \quad \mathrm{R}^{1}$-DIRET $\quad \mathrm{R}^{2}$-pegar

'você disse que (há a potencialidade) eu vou pegar fumo para você'

25. ba na ba a $\varnothing$-mã $\varnothing$-arẽ

$1 \quad$ RLS $12 \quad \mathrm{R}^{1}$-DIRET $\quad \mathrm{R}^{2}$-dizer

dza Ga puru Ø-mã tẽ

IRRLS 2 roça $\mathrm{R}^{2}$-DIRET $\mathrm{ir} / \mathrm{vir}$

'eu disse que você vai para a roça'

\subsection{Verbos de cognição-elocução ('ver', 'saber', 'pensar', 'dizer' etc.)}

Os verbos de cognição-elocução apresentam as seguintes características semânticas e sintáticas, conforme Givón (2001:42):

\section{Características semânticas}

- O verbo da oração principal codifica um evento ou estado mental ou um evento de percepção ou cognição ou um ato de fala.

- O sujeito do verbo é um agente marcado pelo caso dativo.

- O estado ou evento codificado na oração completiva é análogo ao paciente do verbo da oração principal

\section{Características sintáticas}

- Não há restrições quanto à correferencialidade entre a oração principal e a oração completiva.

- Em geral, a oração completiva terá uma oração principal com estrutura finita, com um sujeito plenamente expresso e morfologia verbal finita.

- As duas orações podem ser separadas por um morfema subordinador.

- As duas orações têm contornos intonacionais separados. 


\subsubsection{Construções com o verbo $-m \tilde{u}$ 'ver'}

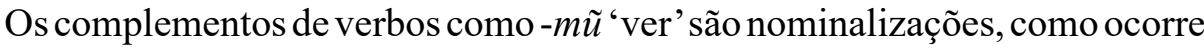
com os complementos de verbos modalidade. As construções nominalizadas nesses casos são precedidas por uma construção oblíqua correferente com o sujeito enfático, que inicia a sentença. Os pronomes dessas construções são da série absolutiva e são regidos pela posposição oblíqua $-j \varepsilon / t \varepsilon$. Semanticamente e estruturalmente, funcionam como objeto direto da oração principal.

26. ba Na ba $\left[\begin{array}{lllll}\mathbf{a} & \varnothing-\mathbf{j} \varepsilon \quad \text { kayã } & \varnothing-\mathbf{b} \tilde{\mathbf{i}}-\mathbf{n}\end{array}\right] \quad$ Ø-mũ

$1 \quad$ RLS $1 \quad 2 \quad \mathrm{R}^{1}$-OBL $\quad$ cobra $\mathrm{R}^{1}$-matar-NLZ $\quad \mathrm{R}^{1}$-ver

'eu vi o matar da cobra por você '(eu vi que você matou a cobra)'

27. ba na ba i $\varnothing$-je $\left[\begin{array}{llll}\mathbf{a} & \varnothing-\mathbf{j} \varepsilon \quad \text { Kayã }\end{array}\right.$

$1 \quad$ RLS $\quad 1 \quad 1 \quad \mathrm{R}^{1}$-OBL $\quad 2 \quad \mathrm{R}^{1}$-OBL $\quad$ Cobra

$\varnothing$-bĩ-n] $\quad \varnothing-$-mũ-j Ket

$\mathbf{R}^{1}$-matar-NLZ $\quad \mathbf{R}^{1}$-ver-NLZ NEG

'não houve o ver de mim do matar da cobra por você'

'(eu não vi que você não matou a cobra)'

28. ba na ba $\varnothing$-omũ

$1 \quad$ RLS $\quad 1 \quad \mathrm{R}^{2}$-ver

a $\varnothing$-je kayã $\varnothing$-bĩ-n] Ket

2 R ${ }^{1}$-OBL cobra $R^{1}$-matar-NLZ NEG

'eu vi que não houve o matar da cobra por você' '(eu vi que você não matou a cobra)'

29. ga na ga [mẽnire kwərə $\mathbf{j}$-are-j]

2 RLS 2 mulher mandioca $\mathrm{R}^{1}$-arrancar-NLZ $\mathrm{R}^{1}$-ver

'você viu que houve o arrancar de mandioca pela mulher'

'(você viu que a mulher arrancou mandioca)'

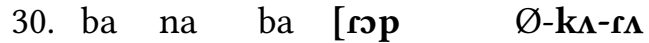

1 RLS 1 cachorro $\mathrm{R}^{1}$-latir-NLZ

Ø-ग Ø-ã-m] $\varnothing$-mũ

ASS.INSTR $\quad \mathrm{R}^{2}$-estar.em.pé-NLZ $\quad \mathrm{R}^{1}$-ver

'eu vi que houve o latido do cachorro' '(eu vi que o cachorro latiu)' 


\subsubsection{O verbo -ma 'saber'}

As construções completivas de cognição-elocução com o verbo -ma 'saber' podem ocorrer justapostas à oração principal, caso que são orações com predicados verbais, ou podem ser nominalizações. Neste último caso, precedem o verbo, formando um constituinte com este, como requerido pela estrutura argumental dos verbos transitivos em Xikrín.

Construções com nominalizações:

31. ba na Ba i $\varnothing$-je $\varnothing$-ma-ri ket

$1 \quad$ RLS $1 \quad 1 \quad 1 \quad \mathrm{R}^{1}$-OBL $\quad \mathrm{R}^{2}$-saber-NLZ NEG

[nũm mẽnire kwərə $\mathbf{j}$-are]

$\mathrm{SD} \quad$ mulher mandioca $\mathrm{R}^{1}$-arrancar

'não há o saber por mim de que a mulher arrancou mandioca'

'(eu não sei que a mulher arrancou mandioca)'

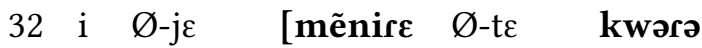

$1 \mathrm{R}^{2}$-OBL Mulher $\mathrm{R}^{1}$-OBL mandioca

j-are-j] $\quad \varnothing$-ma-ri ket

$\mathrm{R}^{1}$-arrancar-NLZ $\mathrm{R}^{1}$-saber-NLZ $\quad$ NEG

'não há o saber por mim do arrancar de mandioca pela mulher'

'(eu não sei que a mulher arrancou mandioca)'

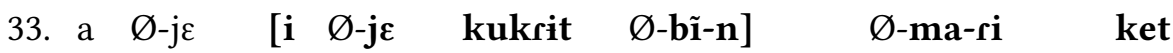

$2 \mathrm{R}^{2}$-OBL $1 \quad \mathrm{R}^{2}$-OBL anta $\quad \mathrm{R}^{1}$-matar-NLZ $\mathrm{R}^{1}$-saber-NLZ NEG 'não há o saber por você de que eu matei anta' '(você não sabe que eu matei anta)'

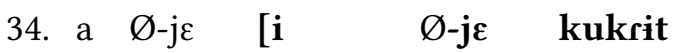

$2 \mathrm{R}^{2}$-OBL 1SG.ABS $\mathrm{R}^{2}$-OBL Anta

Ø-bĩ-n] Ø-ma-ri Ket

$\mathrm{R}^{1}$-matar-NLZ $\mathrm{R}^{1}$-saber-NLZ NEG

'você não sabe que eu matei anta'

'(não há o saber por você do matar de anta por mim)'

Construção com verbo pleno:

35. benadgwərə na ku-ma nũm mẽmi pili Ø-kukwə cacique RLS $\mathrm{R}^{2}$-saber $\mathrm{SD}$ homem Castanha $\mathrm{R}^{1}$-cortar 'o cacique sabe que os homens cortaram castanha' 


\subsection{3 -owagno 'esquecer'}

Em Xikrín, o verbo -əwagno 'esquecer' requer nomes como complementos, de sorte que seus complementos são ou nomes inerentes ou nominalizações.

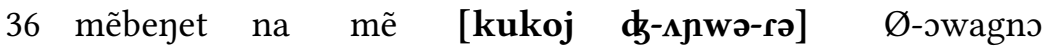
Velho RLS HUM macaco $\mathrm{R}^{1}$-flechar-NLZ $\mathrm{R}^{1}$-esquecer 'o velho esqueceu (de) flechar o macaco'

37. mẽbeyet na Mẽ $\varnothing$-te [kukoj Velho RLS HUM $\mathrm{R}^{2}$-OBL Macaco

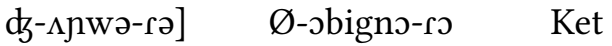

$\mathrm{R}^{1}$-flechar-NLZ $\quad \mathrm{R}^{1}$-esquecer-NLZ NEG

'o velho não esqueceu (de) flechar o macaco'

'(não houve o esquecer do flechar do macaco pelo velho)'

38. mẽnire na mẽ [jat $\mathbf{j}$-are-j] Mulher RLS HUM batata doce $\mathrm{R}^{1}$-arrancar-NLZ $\mathrm{R}^{1}$-esquecer 'a mulher esqueceu (de) arrancar batata doce'

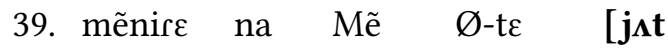

Mulher RLS HUM $\mathrm{R}^{2}$-OBL batata doce

j-are-j] $\quad$ -

$\mathrm{R}^{1}$-arrancar-NLZ $\mathrm{R}^{1}$-esquecer-NLZ NEG

'não houve o esquecer da mulher do arrancar de batata-doce por ela' '(a mulher não esquecer (de) arrancar batata-doce)'

40. ga na ga [i Ø-mã pi?okaprĩ

$\begin{array}{llllll}2 & \text { RLS } & 2 & 1 & \mathrm{R}^{1} \text {-DIRET } & \text { dinheiro }\end{array}$

Ø-jã-rã] $\quad$ Ø-১wagn

$\mathrm{R}^{1}$-dar-NLZ $\mathrm{R}^{1}$-esquece

'você esqueceu (de) me dar dinheiro'

\section{Considerações finais}

Neste estudo descrevemos estratégias usadas em Xikrín para a construção de complementos que correspondem em outras línguas a orações substantivas. Vimos que em Xikrín há dois tipos de construções, uma com verbos plenos e outra com nominalizações. Apenas verbos de manipulação como 'dizer' 
possuem complementos oracionais com núcleo verbal, e o verbo 'saber' pode ter complementos oracionais com verbos plenos em seu núcleo ou nominalizações. Entretanto, mesmo nesses casos, quando o predicado vem seguido de advérbio, como a partícula de negação, a nominalização é exigida. Nos demais casos, como mostramos, não há orações subordinadas substantivas, mas nominalizações.

Esperamos, com este estudo, contribuir para o conhecimento desta língua Jê e estimular trabalhos comparativos sobre o tema aqui enfocado com as línguas dessa família.

\section{Referências}

Dixon, R. M. W. 2010a. Basic linguistic theory. v. 2. Oxford: Oxford University Press. . 2010b. Basic linguistic theory. v. 3. Oxford: Oxford University Press.

Givón, T. 2001. Syntax: a introduction. v. II. Amsterdan: John Benjamins Publishing Press.

Noonan, M. Complementation. In: SHOPEN, Timothy (ed). Language typology and syntactic description. v. 3. Cambridge: Cambridge University Press, 2007.

Rodrigues, A. D. 1986. Línguas brasileiras. Para o conhecimento das línguas Indígenas. São Paulo: Edições Loyola.

Thompson, Sandra A. \& R. Logacre. Adverbial clauses. 1985. In Timothy Shopen (ed). Language typology and syntactic description. v. 2. Cambridge: Cambridge University Press.

Data recebimento: 09/05/2015

Data aceite: $12 / 07 / 2015$ 